\title{
Male-mediated prenatal loss: Functions and mechanisms
}

\author{
${ }^{1}$ Department of Biology, Duke University, Durham, North Carolina \\ ${ }^{2}$ Department of Integrative Biology, Michigan State University, East Lansing, Michigan \\ ${ }^{3}$ Department of Evolutionary Anthropology, Duke University, Durham, North Carolina \\ ${ }^{4}$ Institute of Primate Research, National Museums of Kenya, Nairobi, Kenya \\ ${ }^{5}$ Department of Psychology, University of Michigan, Ann Arbor, Michigan \\ ${ }^{6}$ Department of Anthropology, University of Michigan, Ann Arbor, Michigan
}

Matthew N. Zipple ${ }^{1}$ (1) | Eila K. Roberts ${ }^{2}$ | Susan C. Alberts ${ }^{1,3,4}$ | Jacinta C. Beehner ${ }^{5,6}$

\section{Correspondence}

Matthew N. Zipple, Department of Biology, Duke University, Science Drive, Durham 27708, NC.

Email: matthew.zipple@duke.edu

Funding information

Duke University; Michigan State University; National Institute on Aging, Grant/Award Numbers: P01-AG031719, R01-AG053308; National Science Foundation, Grant/Award Numbers: BCS-0715179, BCS-0824592, IOS1255974, IOS-1456832

\begin{abstract}
Sexually selected infanticide has been the subject of intense empirical and theoretical study for decades; a related phenomenon, male-mediated prenatal loss, has received much less attention in evolutionary studies. Male-mediated prenatal loss occurs when inseminated or pregnant females terminate reproductive effort following exposure to a nonsire male, either through implantation failure or pregnancy termination. Male-mediated prenatal loss encompasses two sub-phenomena: sexually selected feticide and the Bruce effect. In this review, we provide a framework that explains the relationship between feticide and the Bruce effect and describes what is known about the proximate and ultimate mechanisms involved in each. Using a simple model, we demonstrate that male-mediated prenatal loss can provide greater reproductive benefits to males than infanticide. We therefore suggest that, compared to infanticide, malemediated prenatal loss may be more prevalent in mammalian species and may have played a greater role in their social evolution than has previously been documented.
\end{abstract}

\section{KEYWORDS}

Bruce effect, feticide, implantation failure, pregnancy block, pregnancy disruption, pregnancy failure, pregnancy interruption, pregnancy termination, reproductive failure

\section{1 | INTRODUCTION}

Infanticide by males is widely viewed as one of the most extreme forms of sexual conflict whereby males are able to increase their reproductive opportunities by removing unrelated dependent infants. ${ }^{1}$ In numerous species, infanticide thus induces the mother to quickly return to a fertile state, allowing an infanticidal male to mate with her sooner than if he had not intervened. Infanticide has been a focus of study for decades and has been suggested to be involved in the evolution of numerous characteristics of mammalian societies (particularly in primates), including monogamy, promiscuity, synchronous breeding, concealed ovulation, sexual swellings, female-female associations, female-male associations, and small group sizes (reviewed in Palombit ${ }^{1}$ ).

Yet, another phenomenon that induces mothers to return to a fertile state, male-mediated prenatal loss, has received far less attention in evolutionary studies. In this review, we provide an overview of malemediated prenatal loss, a phenomenon by which inseminated or pregnant females abort reproduction following exposure to a nonsire male, either through implantation failure or pregnancy termination. Two related types of male-mediated prenatal loss have been identified (Box 1): (a) Sexually selected feticide occurs when direct aggression from a nonsire male causes a female to terminate an established pregnancy, either by causing direct damage to the fetus and/or by inducing a generalized stress response in the mother. ${ }^{2-6}$ (b) The Bruce effect occurs when females terminate a conceptive event (i.e., implantation failure or pregnancy termination) after exposure to nonsire males without the need for male aggression (although male aggression may occur, aggression is not a necessary component). "Exposure" to nonsire males may be in the form of physical contact with the male himself, exposure to his secretions (i.e., chemosensory exposure), or exposure 


\section{BOX 1 Glossary of terms}

Male-mediated prenatal loss. Male-mediated prenatal loss is any cessation of reproductive effort, either through implantation failure or pregnancy termination that results from a female's exposure to nonsire males. Nonsire males may alter their behavior and/or neuroendocrine secretions in the presence of females to return pregnant females to a fertile state, allowing males to mate with females earlier than they would otherwise. We use the phrase male-mediated prenatal loss (as opposed to male-mediated pregnancy termination or male-mediated implantation failure) because this term includes both possibilities and because natal loss may occur at different times across different species. As we argue, the distinction between male-mediated implantation failure and pregnancy termination is primarily a mechanistic difference, not a functional one; the functional consequence for males and females are nearly equivalent in each case.

Sexually selected feticide occurs when males harass pregnant females with threats and aggression to the extent that females terminate pregnancies. Such harassment (by males or females) has been associated with reproductive failure in a number of mammalian taxa and at all stages of reproduction, including ovulation, conception, pregnancy, and infant survival. ${ }^{31,71}$ Intense harassment can activate the generalized stress response in female victims, ${ }^{95}$ and reproductive failure is presumed to occur in response to this severe environmental stressor. ${ }^{71}$

The Bruce effect occurs when females terminate pregnancies after some form of sensory exposure (olfactory, visual, auditory, or tactile) to nonsire males. Importantly, although nonsire males may exhibit aggression toward females, aggression from males is not necessary to elicit the Bruce effect. Females are even known to seek out sensory input from nonsire males, as when inseminated female mice investigate the scent secretions of novel males. ${ }^{96,97}$

The first clear demonstration of the Bruce effect occurring in a natural rodent population came in 1999 from a study of wild alpine marmots (Marmota marmota), ${ }^{14}$ in which mated females almost universally failed to reproduce after being taken over by a nonsire male. However, after two experimental studies under semi-natural conditions ${ }^{98,99}$ failed to detect a Bruce effect in gray-tailed voles (Microtus canicaudus) and prairie voles (Microtus ochrogaster), some authors argued that the Bruce effect in rodents was a laboratory artifact and therefore had little or no role in rodent social evolution. ${ }^{100,101}$ A recent study in bank voles (Myodes glareolus) ${ }^{78}$ demonstrated that the Bruce effect does occur in semi-natural vole populations, but only under particular social conditions (single female, single male breeding pairs) that were not present in earlier field studies. ${ }^{9899}$ This most recent study in bank voles combined with the earlier study in alpine marmots, refute the arguments of Wolff ${ }^{100}$ and Krebs et al., ${ }^{101}$ and instead provide confirmation that the Bruce effect occurs under both laboratory and natural conditions.

Some have argued that male-mediated prenatal loss does not constitute the Bruce effect unless chemosensory cues are involved. ${ }^{11}$ These authors have opted to use the term Pregnancy block. In our view, this restriction limits the utility of the term "Bruce effect," since it could only be used when researchers had complete knowledge of the mechanism, and it would necessitate a different term for each mechanism involved, resulting in a proliferation of terms for phenomena that have the same functional significance. For instance, primates in particular are known to have "emancipated" themselves from the hormonal control of many processes that are routinely governed by chemosensory input in other mammalian taxa. ${ }^{102,103}$ The shift from chemical cues (e.g., as in most rodents) to visual or auditory cues (e.g., as in many primates) often produces very similar downstream effects (e.g., pregnancy termination); thus, we argue that functionally-equivalent processes should not be considered as separate phenomena with separate terms.

Additionally, several studies in humans have generalized the term "Bruce effect" to refer to spontaneous abortion in response to a range of stressful environments that may threaten offspring survival. ${ }^{19,20,104}$ While severe stress is undoubtedly an important factor in human fertility and reproduction, we discourage the use of the term "Bruce effect" unless pregnancy loss can be attributed to exposure to a nonsire male.

Post-implantation abortion, pregnancy disruption, pregnancy failure, pregnancy interruption. Each of these terms is a more generic form of pregnancy failure. These terms should be used when there is no evidence that the pregnancy loss was male-mediated.

Peri-implantation failure, implantation failure. Each of these terms is a more generic term for failed implantation. These terms should be used when there is no evidence that the implantation failure was male-mediated.

to other sensory stimuli (i.e., visual or acoustic exposure). ${ }^{7-13}$ We argue that, despite the dearth of research effort in the animal literature, malemediated prenatal loss is likely to be more pervasive across mammalian taxa than is infanticide. To draw attention to these studies, we have listed (Supporting Information Table S1) all species (28 species across 17 genera) in which male-mediated prenatal loss has been documented (also available for download at doi:10.5061/dryad.h6980d8). Although this list relies heavily on laboratory studies (e.g., for 15 species of rodents, the Bruce effect has been documented exclusively in the lab), the Bruce effect has been corroborated in field studies of several species. $^{11-15}$

Our main objective is to develop a novel evolutionary framework for understanding male-mediated prenatal loss. In doing so, the article will be partly a review of empirical studies, partly speculative, and partly suggestions for future studies. First, we distinguish between the Bruce effect and feticide by identifying differences in the ultimate and proximate mechanisms involved in each. Second, we extend the anticipated infanticide hypothesis ${ }^{16,17}$ to include feticide prevention 
as a potential explanation for the evolution of the Bruce effect. Third, we hypothesize that, in males, behaviors involved in male-mediated prenatal loss have been under stronger selection than sexually selected infanticide. This hypothesis leads to the prediction that malemediated prenatal loss is more widely prevalent in mammalian taxa than sexually selected infanticide, but is-as yet-undocumented in most species in which it occurs.

The mechanisms underlying male-mediated prenatal loss (especially the Bruce effect) have received a great deal of experimental study in laboratory settings. ${ }^{18}$ By contrast, the evolutionary function of male-mediated prenatal loss has not received an equivalent degree of attention. No doubt this is because male-mediated prenatal loss in natural populations does not typically leave tell-tale signatures (since females often resorb the embryonic/fetal tissue ${ }^{1}$ ) as compared to infanticide (where a dead or missing infant is observed). Moreover, unless research effort is dedicated specifically to identifying malemediated prenatal loss, it can be nearly impossible to detect. Pregnancies, especially early pregnancies, can be difficult to identify, male arrivals can be difficult to track, and reproductive losses can be impossible to link temporally to the arrival of a nonsire male.

Equally problematic, the literature refers to male-mediated prenatal loss by multiple terms that themselves have inconsistent usage. The terms in this review, "feticide" and "Bruce effect," have clear definitions in the literature, yet are often used incorrectly ${ }^{19,20}$ or interchangeably. ${ }^{1,21}$ Other terms, such as pregnancy block, ${ }^{7,22}$ male-induced pregnancy termination, ${ }^{23}$ pregnancy disruption, ${ }^{10,24}$ pregnancy failure, ${ }^{12}$ pregnancy interruption, ${ }^{25}$ and post-implantation abortion ${ }^{26}$ have not been formally defined and are often used differently in different fields. Consequently, although male-mediated prenatal loss is a salient phenomenon for diverse fields-from wildlife biology to evolutionary ecology to human reproductive biology-researchers in these fields may overlook relevant research in other fields because of conflicting terminology.

By promoting a consistent use of terminology and presenting a comprehensive, broad framework, we seek to connect research across different fields and to facilitate hypothesis testing on the different mechanisms and functions of prenatal loss across mammals. Specifically, we review the current state of knowledge on the what, when, how, and why behind male-mediated prenatal loss. For a description of what male-mediated prenatal loss is, see Box 1 , which proposes definitions for the terms that we will use throughout this review. The following sections on when, how, and why focus on both the proximate mechanisms and the evolutionary functions involved.

\section{2 | WHEN CAN MALE-MEDIATED PRENATAL LOSS OCCUR?}

\section{1 | Peri-implantation loss (implantation failure)}

The timing of male-mediated prenatal loss appears to be somewhat species-specific, although it seems to universally occur shortly after exposure to a new male. In numerous rodent species, the mechanisms underlying male-mediated prenatal loss appear to operate only during the peri-implantation period (the period prior to the completion of the implantation process), including mice (Mus musculus ${ }^{7}$ ), deer mice (Peromyscus maniculatus bairdii ${ }^{8}$ ), rats (Rattus norvegicus ${ }^{27}$ ), and Mongolian gerbils (Meriones unguiculatus ${ }^{28}$ ). In these species, the blastocyst is prevented from successful implantation after exposure to a nonsire male without the need for male aggression (see below). After successful implantation, however, laboratory experiments in these species have shown that exposure to a novel male no longer induces pregnancy failure, although it can cause a reduction in investment by females (e.g., smaller pups at birth). ${ }^{29}$ All documented instances of male-mediated implantation failure are more consistent with the Bruce effect than with feticide. We expect that feticide (or, in this case, what would be called embryocide) from male harassment may occur during this peri-implantation period as well, but this distinction in the literature has not yet been made.

\section{2 | Postimplantation loss (pregnancy termination)}

In some rodent species, the Bruce effect is known to occur after successful implantation (e.g., pine vole, Microtus pinetorum ${ }^{26}$; meadow vole, M. pennsylvanicus ${ }^{10}$; prairie vole, M. ochrogaster ${ }^{9}$ ). Notably, in prairie voles, exposure to nonsire males can cause pregnancy termination as late as day 17 in a 24-day gestation period. ${ }^{9}$ The Bruce effect has also been documented after implantation in wild and domestic horses (Equus ferus $^{30}$ and E. caballus ${ }^{11}$ ), domestic dogs (Canis lupus familiaris ${ }^{12}$ ), geladas (Theropithecus gelada ${ }^{13}$ ), and hamadryas baboons (Papio hamadryas ${ }^{15}$ ). Indeed, in a study of wild geladas, one female miscarried following the arrival of a new male only 1 week before her expected parturition date (Beehner, unpublished data). Like the Bruce effect, feticide may also occur any time after implantation, just as severe stressors and/or physical injury can affect the viability of a pregnancy at any time throughout gestation. ${ }^{31}$ Because the literature does not distinguish between the Bruce effect and feticide during the peri-implantation period, we restrict the remainder of our review of feticide to documented cases of fetal loss after implantation has occurred (Supporting Information Table S1; e.g., langurs, Semnopithecus entellus ${ }^{3,4,32}$ and yellow baboons, Papio cynocephalus ${ }^{2,5,6}$ ).

\section{3 | HOW DOES MALE-MEDIATED PRENATAL LOSS OCCUR?}

Thanks to an extensive body of experimental research on the mechanisms of the Bruce effect in rodents (specifically, in mice), we have a very good understanding of how males (and their secretions) mediate loss prior to successful implantation ${ }^{18,33}$ (Figure 1). After successful implantation, however, we have comparatively little knowledge of the mechanisms for any form of male-mediated prenatal loss, particularly for nonrodents. We emphasize that this is an area that needs further research-particularly in primates.

\subsection{Cues received by females from nonsire males}

In mice, the cue from a novel male is primarily (but not entirely) chemosensory. Chemosensory cues include compounds that are transmitted via the vomeronasal organ and/or the main olfactory epithelium. Exposing recently-inseminated female mice to the urine of a 


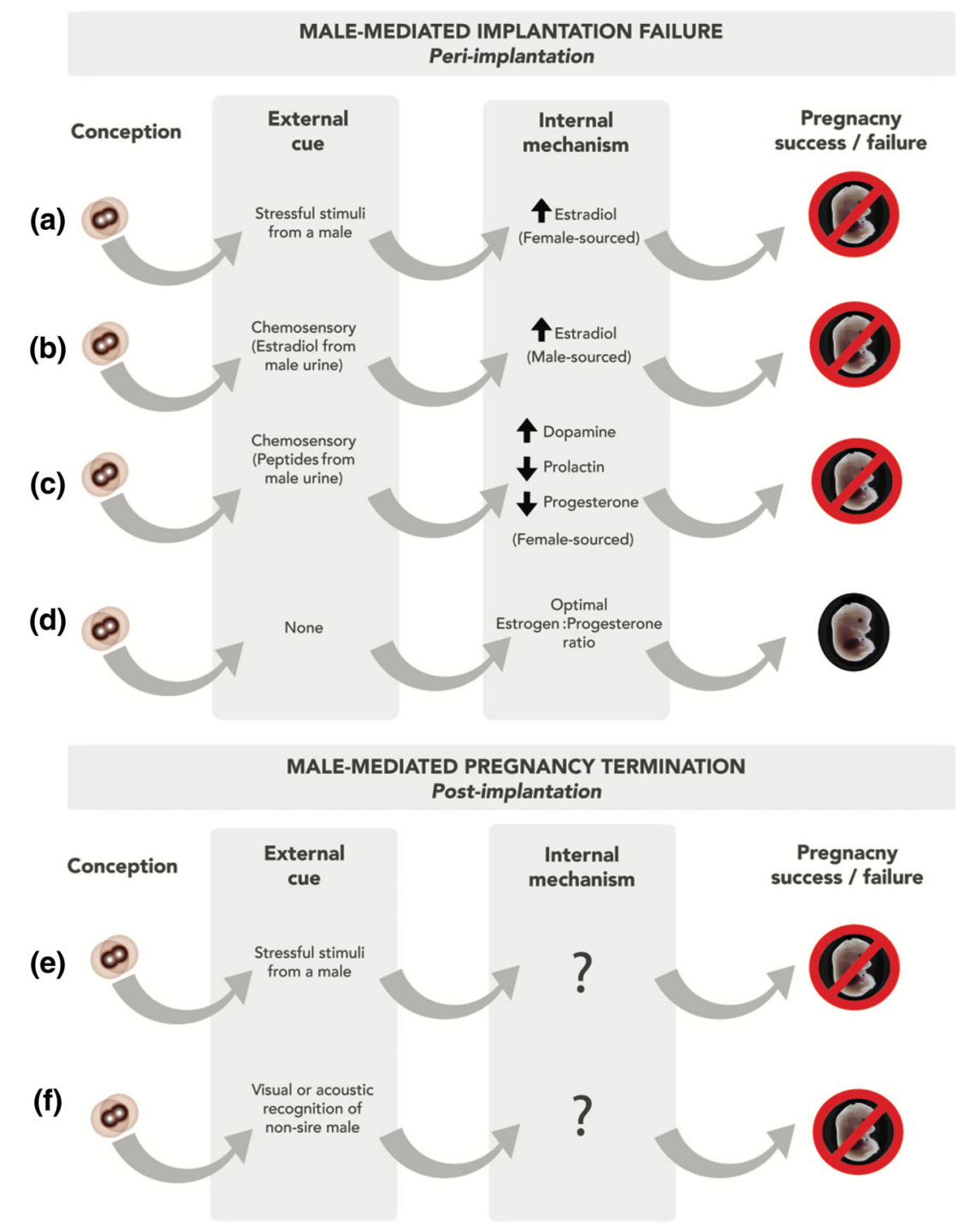

FIGURE 1 Summary of the (nonmutually exclusive) mechanisms involved in male-mediated prenatal loss in mammals. The "external cue" refers to the sensory stimulus that interferes with pregnancy. The "internal mechanism" refers to what we currently know about the neuroendocrine response to the external cue. See text for more details [Color figure can be viewed at wileyonlinelibrary.com]

nonsire male was reported to be both necessary and sufficient for implantation failure, even in the absence of a male. ${ }^{34,35}$ However, another study found that male urine was not sufficient; it had to be combined with direct contact from a nonsire male to ensure implantation disruption. ${ }^{36}$ Indeed, even Hilda Bruce herself (for whom the Bruce effect was named) reported that exposing females to only the urine of novel males was insufficient for pregnancy termination. ${ }^{37}$ Consequently, deCatanzaro and colleagues have suggested that implantation failure in these "urine-only" studies probably resulted from urinary compounds combined with the stress effects of routine handling during the experiments. ${ }^{36}$ Indeed, many chemical mechanisms of reproductive suppression require some degree of auditory or visual reinforcement from the signaler. ${ }^{31}$

In nonrodents, the Bruce effect has been documented in ungulates, carnivores, and primates (Supporting Information Table S1). In these taxa, females need only to experience visual or acoustic exposure to a nonsire male (e.g., across a barrier) with no need for chemosensory cues or direct contact. For example, in domestic horses and dogs, pregnancy termination was more likely to occur when females had visual (but no physical) contact with the nonsire male. ${ }^{11,12}$ We currently do not know whether this difference in sensory cues (chemosensory vs. visual/acoustic) is due to differences across taxa in their dependence on different sensory stimuli or whether it may also relate to the timing of the Bruce effect in these taxa (peri-implantation vs. post-implantation).

\section{2 | Internal mechanisms-peri-implantation}

In rodent models, the two ovarian hormones, estradiol $\left(E_{2}\right)$ and progesterone $\left(\mathrm{P}_{4}\right)$, promote "cross-talk" between the fertilized eggs (i.e., blastocysts) and the uterus to facilitate successful implantation and pregnancy establishment. ${ }^{38}$ Following conception, the blastocysts migrate down the fallopian tube toward the uterus; during this time, $\mathrm{P}_{4}$ primes the uterus to increase $\mathrm{E}_{2}$ receptors, coaxing the uterus into a receptive environment for successful implantation. ${ }^{39,40}$ This window of uterine receptivity depends not just on the presence of $\mathrm{P}_{4}$ and $\mathrm{E}_{2}$, but also on their relative concentrations (Figure $1 \mathrm{~d}$ ): most models in rodents attribute implantation failure to an elevation of the 
$E_{2}: P_{4}$ ratio, either through an increase in $E_{2}$, a decrease in $P_{4}$, or both. Here we list several mechanisms that increase the $E_{2}: P_{4}$ ratio and lead to male-mediated implantation failure. The hypotheses below are not competing but rather complementary pathways that can each produce an elevation of the $E_{2}: P_{4}$ ratio in inseminated female mice, thereby preventing successful implantation.

\section{Endogenous $E_{2}$ from the female disrupts the $E_{2}: P_{4}$ ratio (Figure 1a)}

When females experience severe stressors, the adrenal glands not only increase secretion of "stress" hormones (i.e., glucocorticoids), but also other steroid hormones such as $E_{2}$ (or its precursors). ${ }^{41-43}$ Remarkably, the hormones typically associated with the stress response (i.e., epinephrine and glucocorticoids) have little impact on implantation. ${ }^{44,45}$ Yet, even minute increases in $E_{2}$ associated with adrenal activation can completely disrupt implantation in mice by disrupting the $E_{2}: P_{4}$ ratio. ${ }^{45,46}$ Successful implantation is restored if $P_{4}$ is experimentally elevated ${ }^{42,47}$ and/or if $E_{2}$ is experimentally lowered. ${ }^{48}$ The arrival of a nonsire male may trigger the stress response and subsequent implantation failure in inseminated females.

\section{Exogenous $E_{2}$ from the male disrupts the $E_{2}: P_{4}$ ratio (Figure 1b)}

High concentrations of $E_{2}$ present in the secretions of male rodents may disrupt the $E_{2}: P_{4}$ ratio when they are transferred to females, causing implantation failure. ${ }^{18} \mathrm{~A}$ series of elegant experiments in mice demonstrated that male-sourced $E_{2}$ is readily transferred to females during cohabitation, ${ }^{49}$ particularly during mating. ${ }^{50}$ Male mice that were unable to produce $\mathrm{E}_{2}$ (due to castration ${ }^{51}$ or treatment with an aromatase inhibitor ${ }^{52}$ ) failed to induce the Bruce effect in females with whom they cohabited. Subsequent treatment of these mice with $E_{2}$ injections restored this ability. ${ }^{51}$ Thus, transfer of exogenous $E_{2}$ by nonsire males through cohabitation and/or mating appears to be necessary to induce the Bruce effect in mice.

\section{Downregulation of $P_{4}$ disrupts the $E_{2}: P_{4}$ ratio (Figure $1 c$ )}

Rather than direct upregulation of $E_{2}$, this hypothesis posits that a neuroendocrine cascade indirectly leads to the downregulation of $\mathrm{P}_{4}$. This cascade starts with an increase in hypothalamic dopamine in response to a novel male (or his urine). An increase in dopamine has been found to suppress secretion of the prolactin pulses necessary for maintaining the corpora lutea. ${ }^{53,54}$ In the absence of the corpora lutea, levels of $\mathrm{P}_{4}$ plummet and implantation fails. ${ }^{55}$ In support of this hypothesis, administering exogenous prolactin or $\mathrm{P}_{4}$ to inseminated female rodents rescues pregnancies even after exposure to a novel male. ${ }^{34,56}$

These hypotheses explain how female rodents prevent implantation after exposure to males in general, but how do females prevent implantation after exposure only to nonsire males? The Bruce effect has long been known to occur with a higher probability when novel males are identifiably different from sires (i.e., a different genetic strain $^{35,57,58}$ ), indicating that female discrimination mediates the cue that precipitates pregnancy termination. Under the "olfactory memory hypothesis," female mice form a chemical memory of the siremediated by noradrenaline release in the accessory olfactory bulb ${ }^{59}-$ during a sensitive period immediately after mating. This memory then selectively "gates" any further transmission of the chemosignal to the accessory olfactory bulb, through the medial amygdala, and to the hypothalamus. ${ }^{33}$ In other words, rather than ensuring that novel males do trigger the Bruce effect, the mechanism ensures that familiar males do not trigger it. ${ }^{59}$ The specific peptides that form the basis of this memory are an active area of research (and include peptides related to the major histocompatibility complex, ${ }^{60}$ the exocrine glandsecreting peptide, ${ }^{61}$ and other major urinary peptides ${ }^{62}$ ).

More generally, "recognition" operates via chemosensory modalities, common in rodents ("strain recognition"63) or visual and auditory modalities, common in primates ("individual recognition"64). However, whether the olfactory memory hypothesis (developed for rodents) also applies to visual and auditory "memories," is currently unknown.

\section{3 | Internal mechanisms-Post-implantation}

In a number of different taxa, male-mediated prenatal loss occurs well after successful implantation and appears to occur right up through parturition. Unfortunately, in contrast to the wealth of information on the mechanisms of prenatal loss during the peri-implantation period that we have from laboratory rodents (mainly mice), the mechanisms of prenatal loss during the post-implantation period that are specific to novel male exposure are entirely unknown.

Certainly, evidence from diverse mammalian taxa indicates that external stressors can cause pregnancy failure (or some degree of divestment in offspring) in a number of species. ${ }^{65}$ Similarly, from the biomedical literature we know that severe stress experienced later in pregnancy can lead to pregnancy complications and miscarriage in humans (e.g., pre-eclampsia, gestational hypertension, preterm labor, and low birth weight). ${ }^{66,67}$ Stressful stimuli act via hormonal, neural, and immune pathways to disrupt the processes required for a successful pregnancy, ${ }^{66-68}$ and the mechanisms underlying this stressmediated pregnancy termination are the same ones likely to be involved in male-mediated prenatal loss (Figure 1e). For example, extreme aggression from an immigrant male was associated with elevated glucocorticoids in yellow baboon females and with postimplantation pregnancy termination in some of them ${ }^{5}$; this study cited male harassment as a potent external stressor although it did not confirm this causal link. However, not all cases of observed male-mediated prenatal loss can be explained by extreme stress (Figure 1f). For instance, in wild geladas, pregnancy termination routinely occurs after a new dominant male arrives in a group, even though they rarely exhibit aggression toward females in the group. ${ }^{13}$ We argue that other mechanisms are at work in such cases, although testing this hypothesis will be extremely difficult in wild populations.

\section{4 | WHY DOES MALE-MEDIATED PRENATAL LOSS OCCUR?}

\section{1 | The male perspective}

Male-mediated prenatal loss provides obvious benefits for males. Both sexually selected feticide and the Bruce effect return a female to 
a fertile condition earlier than if male-mediated prenatal loss had not occurred. Given this obvious point, perhaps a more interesting question when considering male-mediated prenatal loss is how does it compare to infanticide as a sexually coercive strategy? Because pregnant females are more temporally distant from a return to cycling than are lactating females, killing a fetus at any given time may increase a male's mating opportunities to a greater extent than would killing an infant. ${ }^{6}$ In other words, the cost of waiting for males that do not commit feticide are much greater than the cost of waiting for males that do not commit infanticide.

To test this claim, we used $\mathrm{R}$ to conduct computer simulations of the behavioral strategies of male yellow baboons (a species in which feticide has been reported $\left.{ }^{2,5,6}\right)$. Specifically, we assessed the number of reproductive opportunities that immigrant males encountered in new social groups under different behavioral strategies: infanticide, feticide, random aggression toward females, and inaction (Box 2). Our simulation results demonstrate that, when faced with an uncertain tenure length, males receive the greatest reproductive benefits from a strategy that flexibly allows them to engage in feticide as well as infanticide. Moreover, males that are restricted to a feticide-only strategy are nevertheless still able to gain greater reproductive benefits than males that are restricted to an infanticide-only strategy (Box 2). The same principle will apply to male-mediated prenatal loss via the Bruce effect, with the added benefit that the Bruce effect is presumably less costly to males than feticide or infanticide because it does not involve engaging in any physical aggression.

Our simulation also demonstrates that feticidal behavior can benefit males even when females display postpartum estrus (i.e., females resume fertility immediately after parturition), a common phenomenon in many mammals. ${ }^{1,69}$ Because infanticidal males derive reproductive benefits by returning noncycling females to fertility, whether sexually selected infanticide occurs in a mammalian taxon is generally predicted by whether females display postpartum amennorhea. ${ }^{69} \mathrm{Sex}$ ually selected infanticide is therefore restricted to a relatively narrow set of mammalian taxa. ${ }^{1}$ While most male mammals benefit from infanticide only when females display postpartum amenorrhea, our simulation demonstrates that males can derive benefits from inducing prenatal loss in females that display postpartum estrus. In light of this finding, we predict that male-mediated prenatal loss may be much more pervasive across the mammalian taxonomy than is infanticide.

\subsection{The female perspective}

The benefits of male-mediated prenatal loss are less obvious for females. From the female's perspective, all forms of male-mediated pregnancy termination impose an immediate cost on females and their reproduction. Both feticide and the Bruce effect cause females to lose a fetus. In addition, feticide (but not the Bruce effect) also necessarily involves the physical cost of severe male aggression. ${ }^{6}$ Yet, we argue that the Bruce effect is nevertheless adaptive for females that find themselves faced with a new male.

Life history theory maintains that pregnant females must weigh the value of each reproductive opportunity across their reproductive lifespan. ${ }^{70}$ Thus, when a female receives a reliable cue that predicts poor reproductive performance, she should divest in reproduction and allocate available resources to self-maintenance. ${ }^{71}$ In response to environmental challenges, mammals have a generalized stress response that diverts energy away from nonessential activities (like reproduction) and toward survival. ${ }^{72}$ Do novel males qualify as a severe external stressor?

Indeed, many mammalian males are hypothesized to exploit this generalized stress response in females in two ways: (a) they inflict physical or psychological stress on females eliciting a stress response (causing feticide), ${ }^{73}$ or (b) they secrete pregnancy-disrupting compounds (mainly, $E_{2}$ found in urine, ${ }^{74}$ axillary perspiration, ${ }^{75}$ or seminal emissions ${ }^{50}$ ) that mimic those excreted by females in response to an external stressor (causing the Bruce effect). ${ }^{65}$ Additionally, as we have seen, the Bruce effect also occurs in pregnant females that have not been exposed to male secretions (e.g., dogs and horses) and do not receive elevated levels of aggression from the new male (e.g., geladas). These latter examples suggest that life history tradeoffs related to the generalized stress response cannot explain all cases of male-mediated prenatal loss.

Perhaps the most cited hypothesis for why male-mediated prenatal loss is adaptive for females is that it saves females the cost of carrying offspring to term that are likely to be killed by an infanticidal male (the "anticipated infanticide" hypothesis ${ }^{16,17}$ ). For instance, female geladas that exhibit the Bruce effect have shorter interbirth intervals than females that experience a common alternative-infant loss ${ }^{13}$-supporting the idea that pregnancy termination is a costmitigating strategy for females when males are predictably infanticidal. By extension, the Bruce effect may be a cost-mitigating strategy for females when males are predictably either feticidal or infanticidal. Terminating pregnancy without having to receive aggression from an otherwise feticidal male would achieve cost-reduction by ending investment in a developing offspring that is doomed while also eliminating the physical costs of enduring a feticidal attack.

Thus, we speculate that the Bruce effect has evolved in species in which infanticide occurs as well as in taxa in which feticide occurred at some point during their evolutionary history. Specifically, the higher the probability of feticide or infanticide by a nonsire male, the more likely selection is to favor female internal mechanisms that actually facilitate male-mediated prenatal loss. Over evolutionary time, females that encounter predictably feticidal and/or infanticidal males will be selected to exhibit neuroendocrine mechanisms that encourage prenatal loss immediately after exposure to these males-even in the absence of aggression. At this stage in this hypothetical evolutionary scenario, males no longer need to be aggressive to reap the benefits of male-mediated prenatal loss. Simultaneously, selection may act in parallel to favor males that are able to induce prenatal loss in females without the need for costly directed aggression, and instead use chemosensory or other signals. ${ }^{18,49,74}$ This may have been the case in mice, where nonsire males are known to upregulate the production of $E_{2}$ in the presence of pregnant females, thus inducing the Bruce effect via transfer of this $E_{2}$ to the females through urine and/or semen. ${ }^{18,49,74}$ We therefore hypothesize that in some cases the Bruce effect may be a derived form of feticide.

From a communications standpoint, the Bruce effect has to be adaptive for females in taxa where it occurs. Unlike feticide or infanticide, the Bruce effect requires the "participation" of female neuroendocrine mechanisms. Chemical signals from a sender (e.g., exogenous 


\section{BOX 2 Relative reproductive benefits of feticide and infanticide for males}

\section{Simulation parameters}

We simulated baboon social groups containing varying numbers of adult females (range 10-50) in all reproductive states (cycling, lactating, pregnant, randomly assigned in proportion to natural occurring frequencies). For each simulation, each female was assigned a wait time based on her reproductive state (0-17 months) ${ }^{105}$ representing the length of time that an immigrant male would have to wait in order to mate with her. Upon immigration, a male was randomly assigned a tenure length, representing the period of time during which he would have a reproductive advantage over other males (uniform distribution, range 1-50 months ${ }^{6}$ ). For each simulated immigration, we ran the simulation five times with the immigrant male following each of the following strategies in one of the simulations:

- Inaction strategy: Do nothing to alter the wait times of females.

- Infanticide strategy: Target the youngest infants, killing up to three (depending on how many are present) and resetting up to three females' wait times to 2 months.

- Feticide strategy: Target pregnant females with the longest wait times (up to three, depending on how many are present), killing fetuses and resetting up to three females' wait times to 2 months.

- Feticide plus strategy: Target both pregnant females and infants, selecting females with the longest wait times (whether pregnant or lactating), and resetting up to three females' wait times to 2 months.

- Random aggression: Attack up to three females at random, resetting each targeted female that was pregnant or lactating to a wait time of 2 months.

We then counted the total number of reproductive opportunities during a male's tenure under each of the five strategies. If a male's tenure lasted long enough for him to reproduce with the same female multiple times, we counted these as multiple reproductive opportunities. Male benefits were therefore cyclical with tenure length, with a period corresponding to the length of the interbirth interval (21 months). We assumed that immigrant males were equally capable of killing any dependent offspring, regardless of its age, and that the costs of infanticide and feticide were equivalent. In fact, feticide may be less costly for males than infanticide if parents are highly protective of neonates. ${ }^{82,106}$ Simulation results

In $96.1 \%$ of 100,000 simulations, the feticide strategy resulted in as many or more conceptive opportunities than inaction, random aggression, or infanticide (Figure 2A). When we then compared the feticide plus strategy with the other four, we found that it returned equal or greater reproductive benefits than any other strategy in $100 \%$ of simulations.

We then altered the parameters to simulate a species that is comparable to baboons in social structure and gestation length, but which displays postpartum estrus (wait times included only a 6-month pregnancy, with no period of postpartum amenorrhea following birth). Under these conditions, the feticide strategy, but not the infanticide strategy, continued to provide males with greater reproductive benefits than inaction (Figure 2b).

Alternative simulation parameters

Under the original simulation parameters, the feticide strategy resulted in worse outcomes than the infanticide strategy only when social groups had three or more infants and fewer than three pregnant females at the time of the male's immigration, limiting the number of fetal targets for immigrant males. Although not common in our simulated baboon groups, this social context would become more common as the $L / G$ ratio is increased. To explore this possibility, we held the total length of lactation plus gestation constant (17 months), but altered the length of each component. For each $L / G$ combination we ran 1,000,000 simulations across the parameter space and computed the average difference in reproductive opportunities between the feticide and infanticide strategy. In cases where the difference between the benefits from feticide and infanticide are greater than zero, feticide should be under stronger selection than infanticide (see Supporting Information Table S2 and Figure S1). We focused here only on the feticide and infanticide strategies, although we should note that the feticide plus strategy would still be superior than either feticide or infanticide under all $L / G$ ratios.

We found that feticide, on average, provided males with greater benefits when the period of lactation was increased even to 14 months ( $G=3$ months, $L / G=4.7$ ). Of the 97 species of primates in the AnAge database ${ }^{107}$ for which both lengths of gestation and age at weaning are available, only chimpanzees have a $L / G$ ratio higher than this value (4.85), indicating that our prediction (i.e., that males are under stronger selection to commit feticide than infanticide) is applicable to the vast majority of primate species on which data is available.

To further explore our parameter space, we removed the 3-target limit that we had previously imposed on the males in our simulation. Removing this constraint on males, which had been imposed based on evidence from yellow baboons, ${ }^{6}$ pushes benefits toward infanticidal males when $L / G$ is greater than 1 . Even under these conditions, which we feel artificially favor infanticide relative to feticide, on average feticide provides greater benefits than infanticide in animals with $L / G$ ratios as high as 2.40 (5 months gestation, 12 months lactation), corresponding to $79 \%$ (77/97) of primates listed in the AnAge database. ${ }^{107}$

Our simulation does not consider all possible social parameters of relevance. For example, it is possible that the degree of female synchrony and the extent male reproductive skew might affect the benefits to males of feticide and infanticide. However, we believe it to be unlikely that these factors will differentially affect the benefits attained from infanticide and feticide, suggesting that their inclusion would be unlikely to qualitatively affect our results. 

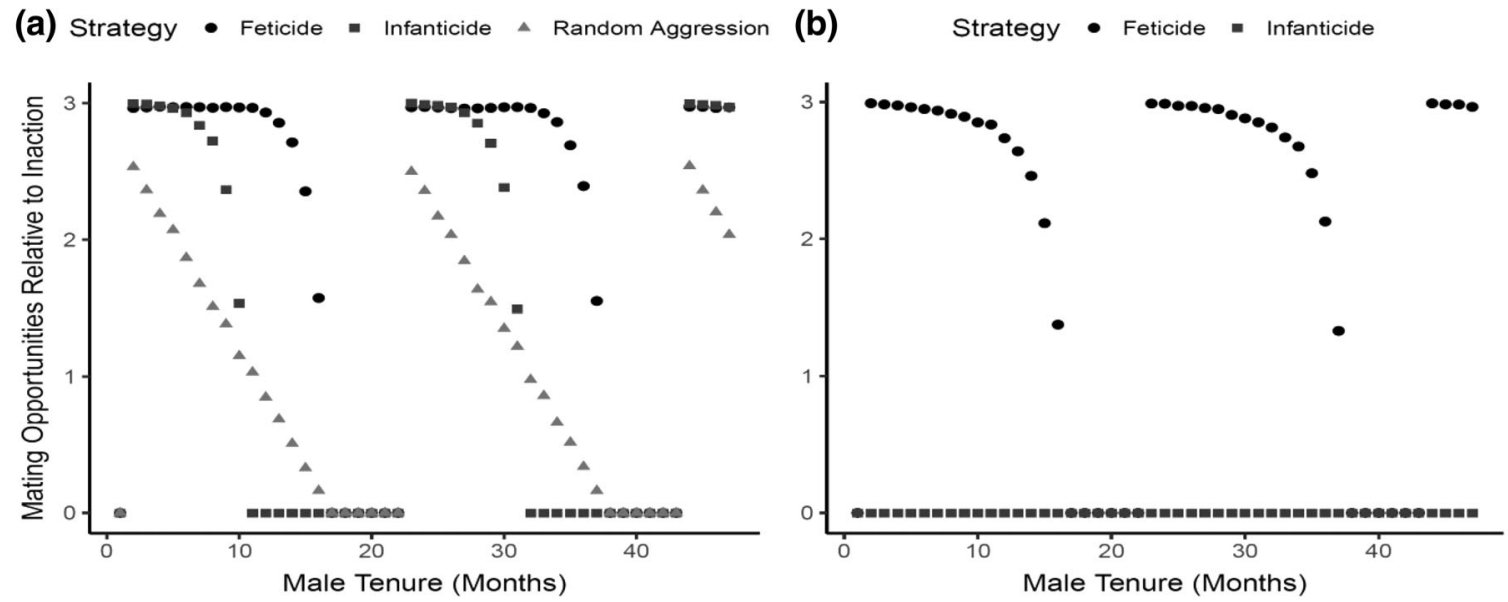

FIGURE 2 (a) Averaged across all group sizes, feticide yields equal or greater reproductive benefits than inaction, random aggression, or infanticide in $96.1 \%$ of simulations. Reproductive benefits attained by each strategy are cyclical with a period matching the 21 -month interbirth interval in the simulation. (b) When females display postpartum estrus, males gain no benefit from infanticide but still benefit from feticide

$E_{2}$ of novel males) are often described in the Bruce effect literature as controlling a receiver (e.g., inducing abortion in a pregnant female). However, this should not be conflated with the sender controlling the fitness of the receiver. This confuses the proximate sense of pheromonal control with the ultimate sense of control over fitness. ${ }^{76}$ Indeed, animal communication systems (even pheromonal ones) are only maintained if communication increases the fitness of both signaler and receiver. ${ }^{77}$ This argument also extends to visual and acoustic signals from a novel male. If the signal is not adaptive for females, selection will favor females that are increasingly less sensitive to it.

If males vary in the probability that they will launch a feticidal or infanticidal attack, females should correspondingly vary the probability of their response. For example, as noted above, female rodents exhibit the Bruce effect in response to the $E_{2}$-laden urine of nonsire males, but not sires, suggesting that females have the capacity to prevent male-sourced $E_{2}$ from triggering a Bruce effect in response to males that do not pose a risk. In addition, pregnant domestic horses ${ }^{11}$ and dogs ${ }^{12}$ are less likely to terminate pregnancy following exposure to a nonsire male if they mate with the male (presumably due to paternity confusion). In meadow voles, pregnancy termination following exposure to nonsire males is less likely in highly aggressive than in less aggressive females, and is more likely after exposure to highly aggressive than to less aggressive males. ${ }^{24}$ Finally, the Bruce effect in bank voles (Myodes glareolus) occurs more frequently in circumstances that may be associated with greater infanticide risk. ${ }^{78}$ Together, these results support the hypothesis that females may express the Bruce effect in a facultative, context-dependent manner. The details of this context-dependence need to be more fully explored within a comparative framework.

\subsection{The balance of male-female conflict}

If the Bruce effect is an adaptive female response to infanticidal males, why do we not observe the Bruce effect in all species with feticidal and/or infanticidal males? First, if feticide/infanticide occurs at very low frequencies (e.g., in yellow baboons ${ }^{6}$ ), then the Bruce effect will confer more costs than benefits on pregnant females. Second, if feticide/infanticide occurs at high frequencies, but the rate of immigration by nonsire males is high relative to the rate of female reproduction, then-again-the Bruce effect will have higher costs than benefits for pregnant females. Females that terminate their pregnancy every time a new alpha male arrives will terminate most of their reproductive efforts. ${ }^{13}$ For example, in one population of chacma baboons (Papio ursinus), infanticide is the leading cause of offspring mortality, ${ }^{79}$ female interbirth intervals are $\sim 2.0$ years, ${ }^{80}$ and male replacement occurs approximately once every 6 months. ${ }^{81}$ Thus, a female chacma baboon may encounter up to four different males during a single interbirth interval. Unsurprisingly, we have no evidence of the Bruce effect in chacma baboons; instead, they exhibit several behavioral counter-strategies to infanticide, including marked behavioral avoidance of nonsire males and friendships with noninfanticidal males, who are sometimes sires. ${ }^{82}$ By contrast, in one population of geladas, where infanticide is also the leading cause of offspring mortality, ${ }^{83}$ male replacement occurs approximately every 3.2 years, ${ }^{84}$ which is longer than the average interbirth interval $\left(2.4\right.$ years $\left.{ }^{85}\right)$. Under these circumstances the Bruce effect is not only observed, but it predominates-with $80 \%$ or more females aborting upon the arrival of a new male. Female geladas that terminate pregnancies via the Bruce effect and conceive with the new male will, on average, have time to wean their offspring prior to the arrival of the next male.

\section{5 | FUTURE DIRECTIONS}

\section{1 | Identifying male-mediated prenatal loss in the wild}

Gathering more data on prenatal loss in wild populations will be critical for testing functional hypotheses. The first step is to get more observations of male-mediated prenatal loss from natural populations. Male-mediated prenatal loss in wild populations can be measured in several ways. First, species-specific study designs can employ demographic data to reveal whether fewer births than expected occur after a novel male enters a group. ${ }^{13,15}$ Additionally, if pregnancies can be determined through visual monitoring, ${ }^{86}$ then one can measure miscarriage rates before versus after the arrival of novel males. ${ }^{6}$ 

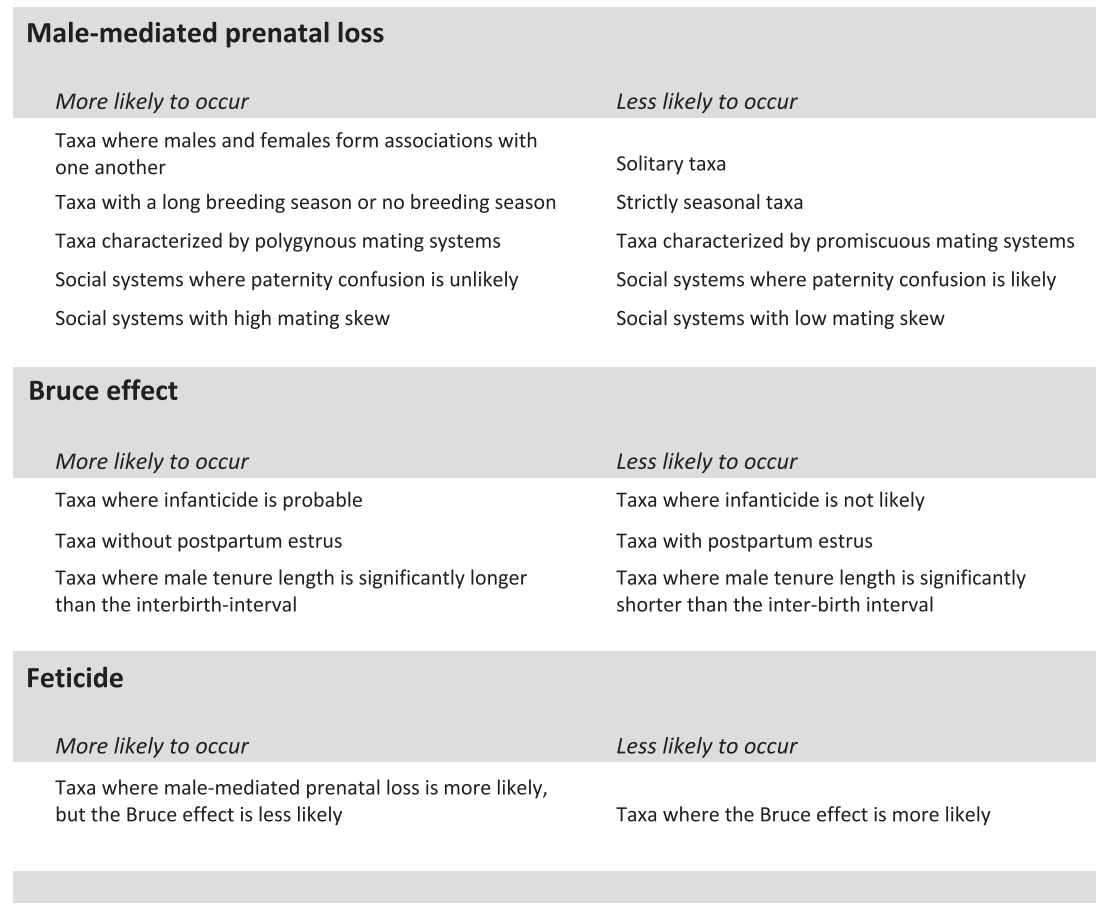

FIGURE 3 Hypothesized relationships between social characteristics of mammalian species and the evolution of male-mediated prenatal loss

When employing demographic data, it is important to control for indirect causes of prenatal loss (e.g., a decline in environmental conditions) that are not caused by the arrival of a nonsire male, but could be coincident with male immigration. For example, Zipple et al. ${ }^{6}$ control for the possibility of environmentally induced prenatal loss in yellow baboons by comparing simultaneous changes in rates of fetal loss following male immigration in groups that experienced male immigration and groups that did not. Because rates of fetal loss increased only in those groups that experienced male immigration, the authors were able to conclude with confidence that the males themselves were causing fetal loss, not any shared environmental factor.

In the absence of a reliable pregnancy sign, researchers have employed a wide variety of methods for determining pregnancy, such as recording ovarian hormones, ${ }^{13}$ body weights, ${ }^{87}$ or other physiological indicators to confirm pregnancy and monitor pregnancy outcomes after the arrival of new males. Finally, to distinguish feticide from the Bruce effect, detailed behavioral observations of male-to-female aggression and/or hormonal measures are required before and after the arrival of a novel male; intense aggression and the elevation of hormones that indicate hypothalamic pituitary adrenal axis activity indicate that females are experiencing physiological stress. A high frequency of pregnancy termination following male replacements in combination with low rates of male-female aggression would support the presence of the Bruce effect, while a low rate of pregnancy termination after male replacements combined with high rates of male-female aggression would support the presence of sexually selected feticide.

\section{2 | Comparative data on male-mediated prenatal loss}

Perhaps the best way to understand how male-mediated prenatal loss evolved, is to place it within a comparative context. Consequently, we encourage studies of male-mediated prenatal loss in diverse mammalian taxa, which will allow researchers to ask new evolutionary questions. ${ }^{88}$ For example, might feticide be an ancestral form of what eventually produced a Bruce effect? If so, what features of a species' social system select for a Bruce effect? We have identified a few in this review (Figure 3), but a more systematic approach to this question is certainly warranted. To answer these questions, phylogenetic comparisons of species that do and do not have male-mediated prenatal loss are needed. However, the data are as yet insufficient to conduct such comparisons. Importantly, to facilitate this comparative approach, researchers need to publish not only when they find evidence for malemediated prenatal loss but also when they find evidence of absence in a particular taxon. We only know of a handful of studies that explicitly state they searched for feticide or the Bruce effect and did not find evidence for it (e.g., rodents ${ }^{89}$; crested macaques [Macaca nigra] ${ }^{90}$ ).

\section{3 | Male-mediated prenatal loss as sexual conflict}

Infanticide is just one type of sexual conflict that "resets" the female reproductive cycle. However, evolutionary models should incorporate all forms of sexual conflict that alter female reproduction to make accurate predictions for both males and females. For example, in taking a phylogenetic comparative approach, recent analyses ${ }^{91-94}$ came to different conclusions about whether infanticide was a causal factor in the evolution of monogamy. If such studies were able to broaden their sexual conflict variable to also include male-mediated prenatal loss, a clearer picture may emerge. At present we have too few empirical data points for this type of comparative analyses. However, once researchers specifically look for it, we expect that male-mediated prenatal loss will be more widespread than the literature currently suggests and that this will open the door to new insights on how sexual conflict and social systems interact. 


\subsection{Identifying the mechanisms that drive male- mediated prenatal loss}

For taxa in which male-mediated prenatal loss is identified, we need to better understand the mechanisms involved. We are well on our way to understanding the mechanisms in mice, where compounds in male urine and semen cause pregnancy termination in the presence of a novel male and pregnancy maintenance in the presence of a sire. However, for many other species, we only know that pregnancy termination is not mediated by a male's urine or any excretion that requires contact. What is the signaling pathway for these taxa? And, how does this signal eventually terminate a pregnancy, sometimes even in the final weeks of pregnancy?

By focusing on these and other questions, we stand to gain insight not only into male-mediated prenatal loss, but into the evolution of sexual conflict more generally. We predict that as research on male-mediated prenatal loss expands, multiple new avenues of research will open up and exciting new discoveries will be made.

\section{ACKNOWLEDGMENTS}

The authors thank our funding sources and institutional support: the National Science Foundation (BCS-0824592, BCS-0715179, IOS1255974 to JCB, IOS-1456832 to SCA), the National Institute on Aging (R01-AG053308 and P01-AG031719 to SCA), Duke University (SCA, MNZ), the University of Michigan (JCB), and Michigan State University (EKR).

\section{ORCID}

Matthew N. Zipple (DD https://orcid.org/0000-0003-3451-2103

\section{REFERENCES}

[1] Palombit RA. 2015. Infanticide as sexual conflict: coevolution of male strategies and female counterstrategies. Cold Spring Harb Perspect Biol 7:1-31.

[2] Pereira ME. 1983. Abortion following the immigration of an adult male baboon (Papio Cynocephalus). Am J Primatol 4:93-98.

[3] Agoramoorthy G, Mohnot SM, Sommer V, Srivastava A. 1988. Abortions in free ranging Hanuman langurs (Presbytis entellus) - a male induced strategy? Hum Evol 3:297-308.

[4] Sommer V. 1994. Infanticide among the langurs of Jodhpur: testing the sexual selection hypothesis with a long-term record. In: Parmigiani S, vom Saal FS, editors. Chur, Switzerland: Infanticide Parent, Care. p 155-198.

[5] Alberts SC et al. 1992. Behavioral, endocrine, and immunological correlates of immigration by an aggressive male into a natural primate group. Horm Behav 26:167-178.

[6] Zipple MN, Grady JH, Gordon JB, et al. 2017. Conditional fetal and infant killing by male baboons. Proc R Soc B Biol Sci 284:20162561.

[7] Bruce HM. 1959. An exteroreceptive block to pregnancy in the mouse. Nature 184:105.

[8] Bronson F, Eleftheriou B. 1963. Influence of strange males on implantation in the deermouse. Gen Comp Endocrinol 3:515-518.

[9] Stehn RA, Richmond ME. 1975. Male-induced pregnancy termination in the prairie vole, Microtus ochrogaster. Science 187: 1211-1213.

[10] Kenney AM et al. 1977. Postimplantation pregnancy disruption in Microtus ochrogaster, M. pennsylvanicus and Peromyscus maniculatus. Reproduction 49:365-367.
[11] Bartoš L, Bartošová J, Pluháček J, Šindelářová J. 2011. Promiscuous behaviour disrupts pregnancy block in domestic horse mares. Behav Ecol Sociobiol 65:1567-1572.

[12] Bartos L et al. 2016. A sociobiological origin of pregnancy failure in domestic dogs. Sci Rep 6:22188.

[13] Roberts EK, Lu A, Bergman TJ, Beehner JC. 2012. A Bruce effect in wild geladas. Science 335:1222-1225.

[14] Hacklander K, Arnold W. 1999. Male-caused failure of female reproduction and its adaptive value in alpine marmots (Marmota marmota). Behav Ecol 10:592-597.

[15] Amann AL, Pines M, Swedell L. 2017. Contexts and consequences of takeovers in hamadryas baboons: female parity, reproductive state, and observational evidence of pregnancy loss. Am J Primato 79:e22649.

[16] Labov JB. 1981. Pregnancy blocking in rodents: adaptive advantages for females. Am Nat 118:361-371.

[17] Rülicke T et al. 2006. Early maternal investment in mice: no evidence for compatible-genes sexual selection despite hybrid vigor. J Evol Biol 19:922-928.

[18] deCatanzaro D. 2015. Sex steroids as pheromones in mammals: the exceptional role of estradiol. Horm Behav 68:103-116.

[19] Saxton KB, Gemmill A, Catalano RA. 2017. Reproductive suppression follows threats to child survival. J Evol Biol 30:889-897.

[20] Catalano RA et al. 2016. Twinning in Norway following the Oslo massacre: evidence of a 'Bruce effect' in humans. Twin Res Hum Genet 19:485-491.

[21] Palombit RA. 2014. Sexual conflict in nonhuman primates. In: Advances in the Study of Behavior, Vol 46, 1st ed. Oxford, UK: Elsevier Inc.

[22] Bruce HM. 1960. A block to pregnancy in the mouse caused by proximity of strange males. J Reprod Fertil 1:96-103.

[23] Storey AE. 1986. Influence of sires on male-induced pregnancy disruptions in meadow voles (Microtus pennsylvanicus) differs with stage of pregnancy. J Comp Psychol 100:15-20.

[24] Storey A, Snow D. 1990. Postimplantation pregnancy disruptions in meadow voles: relationship to variation in male sexual and aggressive behavior. Physiol Behav Elsevier 47:19-25.

[25] Smale L. 1988. Influence of male gonadal hormones and familiarity on pregnancy interruption in prairie voles. Biol Reprod 39:28-31.

[26] Schadler MH. 1981. Postimplantation abortion in pine voles (Microtus pinetorum) induced by strange males and pheromones of strange males. Biol Reprod 25:295-297.

[27] Marashi V, Rülicke T. 2012. The Bruce effect in Norway rats. Biol Reprod 86:1-5.

[28] Rohrbach C. 1982. Investigation of the Bruce effect in the Mongolian gerbil (Meriones unguiculatus). J Reprod Fertil 65:411-417.

[29] Gale T et al. 2013. Exposure to a novel male during late pregnancy influences subsequent growth of offspring during lactation. J Evol Biol Wiley/Blackwell (10.1111) 26:2057-2062.

[30] Berger J. 1983. Induced abortion and social factors in wild horses. Nature 303:59-61.

[31] Beehner JC, Lu A. 2013. Reproductive suppression in female primates: a review. Evol Anthropol 22:226-238.

[32] Sommer V. 1987. Infanticide among free-ranging langurs (Presbytis entellus) at Jodhpur (Rajasthan/India): recent observations and a reconsideration of hypotheses. Primates 28:163-197.

[33] Brennan P, Keverne EB. 2015. Biological complexity and adaptability of simple mammalian olfactory memory systems. Neurosci Biobehav Rev 50:29-40.

[34] Dominic CJ. 1966. Observations on the reproductive pheromones of mice. J Reprod Fertil 11:407-414.

[35] Parkes AS, Bruce HM. 1962. Pregnancy-block in female mice placed in boxes soiled by males. J Reprod Fertil 4:303-308.

[36] deCatanzaro D, Wyngaarden P, Griffiths J, Ham M, Hancox J, Brain D. 1995. Interactions of contact, odor cues, and androgens in strange-male-induced early pregnancy disruptions in mice (Mus musculus). J Comp Psychol 109:115-122.

[37] Bruce H. 1960. Further observations on pregnancy block in mice caused by the proximity of strange males. J Reprod Fertil 1: 311-312. 
[38] Paria BC, Reese J, Das SK, Dey SK. 2002. Deciphering the cross-talk of implantation: advances and challenges. Science 296:2185-2188.

[39] Martel D, Psychoyos A. 1982. Different responses of rat endometrial epithelium and stroma to induction of oestradiol binding sites by progesterone. J Reprod Fertil 64:387-389.

[40] Carson DD, Bagchi I, Dey SK, et al. 2000. Embryo implantation. Dev Biol 223:217-237.

[41] MacNiven E, deCatanzaro D, Younglai EV. 1992. Chronic stress increases estrogen and other steroids in inseminated rats. Physiol Behav 52:159-162.

[42] Thorpe JB, Burgess PS, Sadkowski M, deCatanzaro D. 2013. Estrogen-progesterone balance in the context of blastocyst implantation failure induced by predator stress. Psychoneuroendocrinology 38: 3048-3056.

[43] Thorpe J, Gould KE, Borman ED, deCatanzaro D. 2014. Circulating and urinary adrenal corticosterone, progesterone, and estradiol in response to acute stress in female mice (Mus musculus). Horm Metab Res 46:211-218.

[44] deCatanzaro D, Graham C. 1992. Influences of exogenous epinephrine on two reproductive parameters in female mice: disruption of receptivity but not implantation. Horm Behav 26:330-338.

[45] de Catanzaro D, MacNiven E, Ricciuti F. 1991. Comparison of the adverse effects of adrenal and ovarian seteroids on early pregnancy in mice. Psychoneuroendocrinology 16:525-536.

[46] Ma W, Song H, Das SK, Paria BC, Dey SK. 2003. Estrogen is a critical determinant that specifies the duration of the window of uterine receptivity for implantation. Proc Natl Acad Sci 100:2963-2968.

[47] Macniven E, de Catanzaro D. 1990. Reversal of stress-induced pregnancy blocks in mice by progesterone and metyrapone. Physiol Behav Elsevier 47:443-448.

[48] deCatanzaro D, Macniven E, Goodison T, Richardson D. 1994. Estrogen antibodies reduce vulnerability to stress-induced failure of intrauterine implantation in inseminated mice. Physiol Behav 55: 35-38.

[49] Guzzo AC, Jheon J, Imtiaz F, deCatanzaro D. 2012. Oestradiol transmission from males to females in the context of the Bruce and Vandenbergh effects in mice (Mus musculus). Reproduction 143: 539-548.

[50] deCatanzaro D, Pollock T. 2016. Absorption and distribution of estradiol from male seminal emissions during mating. J Endocrinol 231:245-257.

[51] Thorpe JB, de Catanzaro D. 2012. Oestradiol treatment restores the capacity of castrated males to induce both the Vandenbergh and the Bruce effects in mice (Mus musculus). Reproduction 143: 123-132.

[52] Beaton EA, de Catanzaro D. 2005. Novel males' capacity to disrupt early pregnancy in mice (Mus musculus) is attenuated via a chronic reduction of males' urinary $17 \beta$-estradiol. Psychoneuroendocrinology 30:688-697.

[53] Li CS, Kaba H, Saito H, Seto K. 1990. Neural mechanisms underlying the action of primer pheromones in mice. Neuroscience 36: 773-778.

[54] Marchlewska-Koj A, Jemiolo B. 1978. Evidence for the involvement of dopaminergic neurons in the pregnancy block effect. Neuroendocrinology 26:186-192.

[55] Bruce H, Parkes A. 1960. Hormonal factors in exteroceptive block to pregnancy in mice. J Endocrinol 20:xxix-xxx.

[56] Rajendren G, Dominic CJ. 1987. The male-induced pregnancy block (the Bruce effect) in mice: re-evaluation of the ability of exogenous progesterone in preventing implantation failure. Exp Clin Endocrinol 89:188-196.

[57] Marsden HM, Bronson FH. 1965. Strange male block to pregnancy: its absence in inbred mouse strains. Nature 207:878-878.

[58] Spironello E, DeCatanzaro D. 1999. Sexual satiety diminishes the capacity of novel males to disrupt early pregnancy in inseminated female mice (Mus musculus). J Comp Psychol 113:218-222.

[59] Keverne EB, de la Riva C. 1982. Pheromones in mice: reciprocal interaction between the nose and brain. Nature 296:148-150.

[60] Leinders-Zufall T et al. 2004. MHC class I peptides as chemosensory signals in the vomeronasal organ. Science 306:1033-1037.
[61] Hattori T, Osakada T, Masaoka T, et al. 2017. Exocrine glandsecreting peptide 1 is a key chemosensory signal responsible for the Bruce effect in mice. Curr Biol 27:3197-3201.e3.

[62] Petrulis A. 2013. Chemosignals, hormones and mammalian reproduction. Horm Behav 63:723-741.

[63] Brennan P, Kaba H, Keverne E. 1990. Olfactory recognition: a simple memory system. Science 250:1223-1226.

[64] Perrett DI, Mistlin AJ, Chitty AJ. 1987. Visual neurones responsive to faces. Trends Neurosci 10:358-364.

[65] de Catanzaro D, Macniven E. 1992. Psychogenic pregnancy disruptions in mammals. Neurosci Biobehav Rev 16:43-53.

[66] Arck PC. 2001. Stress and pregnancy loss: role of immune mediators, hormones and neurotransmitters. Am J Reprod Immunol 46: 117-123.

[67] Christian LM. 2012. Physiological reactivity to psychological stress in human pregnancy: current knowledge and future directions. Prog Neurobiol 99:106-116.

[68] Clark DA. 2008. Immunological factors in pregnancy wastage: fact or fiction. Am J Reprod Immunol 59:277-300.

[69] van Schaik CP. 2000. Social counterstrategies against male infanticide in primates and other mammals. In: Kappeler PM, editor. Primate males: causes and consequences of variation in group composition, Cambridge: Cambridge University Press. p 34-52.

[70] Stearns SC. 1992. The evolution of life histories, Oxford: Oxford University Press.

[71] Wasser SK, Barash DP. 1983. Reproductive suppression among female mammals: implications for biomedicine and sexual selection theory. Q Rev Biol 58:513-538.

[72] Wingfield JC, Sapolsky RM. 2003. Reproduction and resistance to stress: when and how. J Neuroendocrinol 15:711-724.

[73] Smuts BB, Smuts RW. 1993. Male aggression and sexual coercion of females in nonhuman primates and other mammals: evidence and theoretical implications. Adv Study Behav Academic Press 22:1-63.

[74] DeCatanzaro $D$ et al. 2009. Exposure to developing females induces polyuria, polydipsia, and altered urinary levels of creatinine, $17 \beta-$ estradiol, and testosterone in adult male mice (Mus musculus). Horm Behav 55:240-247.

[75] Muir C, Spironello-Vella E, Pisani N, deCatanzaro D. 2001. Enzyme immunoassay of $17 \beta$-estradiol, estrone conjugates, and testosterone in urinary and fecal samples from male and female mice. Horm Metab Res 33:653-658.

[76] Peso $M$ et al. 2015. Pheromonal control: reconciling physiological mechanism with signalling theory. Biol Rev Wiley/Blackwell (10.1111) 90:542-559.

[77] Searcy WA, Nowicki S. 2005. The evolution of animal communication: reliability and deception in signaling systems, Princeton, NJ: Princeton University Press.

[78] Eccard JA, Dammhahn M, Ylönen H. 2017. The Bruce effect revisited: is pregnancy termination in female rodents an adaptation to ensure breeding success after male turnover in low densities? Oecologia 185:81-94.

[79] Cheney DL, Seyfarth RM, Fischer J, et al. 2006. Reproduction, mortality, and female reproductive success in chacma baboons of the Okavango Delta, Botswana. In: Swedell L, Leigh S, editors. Reproduction and fitness in baboons: behavioral, ecological, and life history perspectives, New York: Springer. p 147-176.

[80] Cheney D et al. 2004. Factors affecting reproduction and mortality among baboons in the Okavango Delta, Botswana. Int J Primatol 25: 401-428.

[81] Hamilton W, Bulger J. 1990. Natal male baboon rank rises and successful challenges to resident alpha males. Behav Ecol Sociobiol 26: 357-362.

[82] Palombit RA et al. 1997. The adaptive value of "friendships" to female baboons: experimental and observational evidence. Anim Behav 54:599-614.

[83] Beehner JC, Bergman TJ. 2008. Infant mortality following male takeovers in wild geladas. Am J Primatol 70:1152-1159.

[84] Snyder-Mackler N, Alberts SC, Bergman TJ. 2012. Concessions of an alpha male? Cooperative defence and shared reproduction in multi-male primate groups. Proc Biol Sci 279:3788-3795. 
[85] Roberts EK, Lu A, Bergman TJ, Beehner JC. 2017. Female reproductive parameters in wild geladas (Theropithecus gelada). Int J Primatol 38:1-20.

[86] Altmann SA, Altmann J. 1973. Baboon ecology: African field research. Chicago, Illinois: University of Chicago Press.

[87] Young AJ, Carlson AA, Monfort SL, Russell AF, Bennett NC, Clutton-Brock T. 2006. Stress and the suppression of subordinate reproduction in cooperatively breeding meerkats. Proc Natl Acad Sci 103:12005-12010.

[88] Nunn C. 2011. The comparative method in evolutionary anthropology and biology. Chicago, Illinois: University of Chicago Press.

[89] Blumstein D. 2000. The evolution of infanticide in rodents: a comparative analysis. In: van Schaik $\mathrm{CP}$, Janson $\mathrm{CH}$, editors. Infanticide by males its Implic, Cambridge, UK: Cambridge University Press, p 178-197.

[90] Kerhoas D, Perwitasari-Farajallah D, Agil M, Widdig A, Engelhardt A. 2014. Social and ecological factors influencing offspring survival in wild macaques. Behav Ecol 25:1164-1172.

[91] Lukas D, Clutton-Brock TH. 2013. The evolution of social monogamy in mammals. Science 341:526-530.

[92] Opie C, Atkinson QD, Dunbar RIM, Shultz S. 2013. Male infanticide leads to social monogamy in primates. Proc Natl Acad Sci 110: 13328-13332.

[93] Lukas D, Huchard E. 2014. The evolution of infanticide by males in mammalian societies. Science 346:841-844.

[94] Opie C, Atkinson QD, Dunbar RIM, Shultz S. 2014. Reply to Lukas and Clutton-Brock: infanticide still drives primate monogamy. Proc Natl Acad Sci 111:E1675-E1675.

[95] Sapolsky RM, Romero LM, Munck AU. 2000. How do glucocorticoids influence stress responses? Integrating permissives, suppressive, stimulatory, and preparative actions. Endocr Rev 21 55-89.

[96] DeCatanzaro D, Murji T. 2004. Inseminated female mice (Mus musculus) investigate rather than avoid novel males that disrupt pregnancy, but sires protect pregnancy. J Comp Psychol 118: 251-257.

[97] Becker SD, Hurst JL. 2009. Female behaviour plays a critical role in controlling murine pregnancy block. Proceed Biol Sci 276: 1723-1729.

[98] de la Maza HM, Wolff JO, Lindsey A. 1999. Exposure to strange adults does not cause pregnancy disruption or infanticide in the gray-tailed vole. Behav Ecol Sociobiol Springer-Verlag. 45: 107-113.

[99] Mahady S, Wolff J. 2002. A field test of the Bruce effect in the monogamous prairie vole (Microtus ochrogaster). Behav Ecol Sociobiol Springer-Verlag 52:31-37.

[100] Wolff JO. 2003. Laboratory studies with rodents: facts or artifacts? Bioscience Oxford University Press 53:421-427.

[101] Krebs $C$ et al. 2007. Social behaviour and self-regulation in murid rodents. In: Wolff JO, Sherman PW, editors. Rodent societies : An Ecological and Evolutionary Perspective, Chicago: University of Chicago Press. p 173-181.

[102] Keverne EB et al. 1996. Primate brain evolution: genetic and functional considerations. Proceed Biol Sci 263:689-696.
[103] Wallen K, Zehr JL. 2004. Hormones and history: the evolution and development of primate female sexuality. J Sex Res 41:101-112.

[104] Catalano R et al. 2017. Separating the Bruce and Trivers-Willard effects in theory and in human data. Am J Hum Biol 30:e23074.

[105] Gesquiere LR, Altmann J, Archie EA, Alberts SC. 2018. Interbirth intervals in wild baboons: environmental predictors and hormonal correlates. Am J Phys Anthropol 166:107-126.

[106] Nguyen N, van Horn RC, Alberts SC, Altmann J. 2009. "Friendships" between new mothers and adult males: adaptive benefits and determinants in wild baboons (Papio cynocephalus). Behav Ecol Sociobiol 63:1331-1344.

[107] de Magalhaes JP, Costa J. 2009. A database of vertebrate longevity records and their relation to other life-history traits. J Evol Biol Wiley/Blackwell (10.1111) 22:1770-1774.

\section{AUTHOR BIOGRAPHIES}

Matthew N. Zipple is a PhD candidate in the biology department at Duke University. His research focuses on the ecology and evolution of social behavior, life history strategies, and communication in primate and nonprimate animals.

EILA K. Roberts is an Academic Specialist in the Department of Integrative Biology at Michigan State University. Her research focuses on the development and function of female social behavior and reproduction.

Susan C. Alberts is The Robert F. Durden Professor of Biology and Evolutionary Anthropology, and Chair of the Evolutionary Anthropology Department, at Duke University. She studies the behavior, ecology, physiology, and genetics of wild populations of large mammals, particularly the baboons of the Amboseli basin in southern Kenya.

Jacinta C. Beehner is an Associate Professor in the Departments of Anthropology and Psychology at the University of Michigan. She is broadly interested in the interactions between hormones and animal behavior, with a particular focus on reproductive strategies, sexual conflict, and reproductive suppression in wild primates.

\section{SUPPORTING INFORMATION}

Additional supporting information may be found online in the Supporting Information section at the end of this article.

How to cite this article: Zipple MN, Roberts EK, Alberts SC, Beehner JC. Male-mediated prenatal loss: Functions and mechanisms. Evolutionary Anthropology. 2019;28:114-125. https://doi.org/10.1002/evan.21776 